\title{
Fully solvable mathematical scheme in finding out the right mass and width values of $f_{0}(500)$ and $\rho^{0}(770)$ mesons
}

\author{
Stanislav Dubnicka ${ }^{1 *}$ and Anna Zuzana Dubnickova ${ }^{2}$ \\ ${ }^{1}$ Institute of Physics, Slovak Academy of Sciences, Bratislava, Slovakia \\ ${ }^{2}$ Department of Theoretical Physics, Comenius University, Bratislava, Slovakia
}

\begin{abstract}
Starting from the phase representations with one subtraction of the pion scalar-isoscalar and vector-isovector charged pion electromagnetic form factor and exploiting the most accurate information on the S-wave isoscalar and the P-wave isovector $\pi \pi$ scattering phase shifts, respectively, to be obtained from the existing inaccurate experimental data by means of the GarciaKamiński-Peláez-Yndurain Roy-like equations with an imposed crossing symmetry condition, in the framework of the so-called "fully solvable mathematical scheme" the most reliable values of the $f_{0}(500)$ and $\rho^{0}(770)$ meson mass and width are found.
\end{abstract}

\section{Introduction}

If there is a function $F(t)$ to be analytic in the whole complex $t$-plane besides the cuts on the positive real axis from the lowest square root branch point $t=4$ to $+\infty$, fulfills the elastic unitarity condition $\operatorname{Im} F(t)=F(t) e^{-i \delta} \sin \delta$ in elastic region with $\delta$ to be some of the $\pi \pi$ scattering phase shifts, the asymptotic behavior $F(t)_{|t| \rightarrow \infty} \sim 1 / t$, the reality condition $F^{*}(t)=F\left(t^{*}\right)$ and it is normalized at $t=0$ to one, then one can write down using the Cauchy formula a dispersion relation with one subtraction at the point $t=0$, which together with the unitarity condition through the Omnés-Muskelishvili integral equation leads to the phase representation of $F(t)$

$$
F(t)=P_{n}(t) \exp \left[\frac{t}{\pi} \int_{4}^{\infty} \frac{\delta\left(t^{\prime}\right)}{t^{\prime}\left(t^{\prime}-t\right)} d t^{\prime}\right]
$$

to be the starting point for our further investigations.

Under the "fully solvable mathematical scheme" [1] it is understood a procedure leading to a very simple form of $F(t)$ in the variable

$$
q=[(t-4) / 4]^{1 / 2}
$$

by means of an explicit calculation of the integral in the phase representation (1).

The function $F(t)$ on the positive real axis for $t>4$ is complex with the phase $\delta_{F}$ to be defined by the relation

$$
\tan \delta_{F}=\frac{\operatorname{Im} F(t)}{\operatorname{Re} F(t)},
$$

\footnotetext{
*e-mail: fyzidubn@savba.sk
} 
which, however, due to the elastic unitarity condition, phenomenologically verified to be valid approximately up to $1 \mathrm{GeV}$, is identical with the $\pi \pi$ scattering phase shift $\delta$.

Since the transformation (2) is in fact a conformal mapping of the two-sheeted Riemann surface in $t$ variable into one $q$-plane, elastic cut, generated by the square root branch point $t=4$, disappears.

Noticing the conformal mapping (2) in more detail, the first Riemann sheet in $t$ variable, containing only branch points corresponding to opening various thresholds and zeros of $F(t)$, is mapped on the upper half $q$-plane, whereby position of the branch point $t=4$ and the normalization point $t=0$ are mapped into $q=0$ and $q=+i$, respectively, and the real axis from $-\infty$ up to $t=4$, on which $F(t)$ is a real function, is mapped on the whole positive imaginary axis of the $q$-plane.

The second Riemann sheet in $t$ variable, containing branch points, again some zeros and also complex conjugate pairs of poles, which control the shape of $F(t)$, is mapped on the lower half $q$-plane.

If we restrict ourselves only to the elastic region and neglect contributions to $F(t)$ of all inelastic branch points beyond $1 \mathrm{GeV}$, then there are only zeros in the upper and lower half $q$-plane to be accounted as roots of a polynomial in numerator and conjugate pairs of poles according to the imaginary axis exclusively in the lower half $q$-plane to be accounted as roots of a polynomial in denominator of $F(t)$.

As a result one can represent $F(t)$ in the form of the following rational function

$$
F[t(q)]=\frac{\sum_{n=0}^{M} a_{n} q^{n}}{\sum_{r=0}^{N} b_{r} q^{r}} .
$$

Multiplying the numerator and denominator by the complex conjugate denominator, Eq. (4) is changed to the form

$$
F[t(q)]=\frac{\sum_{s=0}^{M+N} c_{s} q^{s}}{\left(\sum_{r=0}^{N} b_{r} q^{r}\right)\left(\sum_{r=0}^{N} b_{r} q^{r}\right)^{*}} .
$$

The reality condition $F^{*}(t)=F\left(t^{*}\right)$ results in the reality of (5) on the positive imaginary axis of the $q$-plane. Then it is straightforward to see that the expression

$$
F[t(q)]=\frac{\left(c_{0}+c_{2} q^{2}+c_{4} q^{4}+\ldots\right)+i\left(c_{1} q+c_{3} q^{3}+c_{5} q^{5}+\ldots\right)}{\left(\sum_{r=0}^{N} b_{r} q^{r}\right)\left(\sum_{r=0}^{N} b_{r} q^{r}\right)^{*}}
$$

actually fulfils the latter claim and through the definition of the phase of $F(t)$ leads to the following parametrization of the $\pi \pi$ scattering phase shift $\delta$

$$
\delta=\arctan \frac{A_{1} q+A_{3} q^{3}+A_{5} q^{5}+A_{7} q^{7}+\ldots}{1+A_{2} q^{2}+A_{4} q^{4}+A_{6} q^{6}+\ldots},
$$

with all coefficients to be real. This parametrization is derived directly from the basic principles like analyticity, unitarity and reality condition.

\section{Pion scalar-isoscalar form factor and $f_{0}(500)$ meson parameters}

The pion scalar-isoscalar form factor $(\mathrm{FF}) \Gamma_{\pi}(t)$ is defined by the matrix element of the scalar quark density

$$
\left\langle\pi^{i}\left(p_{2}\right)|\hat{m}(\bar{u} u+\bar{d} d)| \pi^{j}\left(p_{1}\right)\right\rangle=\delta^{i j} \Gamma_{\pi}(t),
$$


with $t=\left(p_{2}-p_{1}\right)^{2}$ and $\hat{m}=\frac{1}{2}\left(m_{u}+m_{d}\right)$, and fulfils all properties of the function $F(t)$ defined in Sect. 1. Even the normalization of physically nonmeasurable pion scalar $\mathrm{FF} \Gamma_{\pi}(t)$ is equal to one as it is seen from the calculated pion sigma term value with error

$$
\Gamma_{\pi}(0)=(0.99 \pm 0.02) m_{\pi}^{2}
$$

in the framework of the $\chi \mathrm{PT}[2]$, if the pion mass $m_{\pi}$ is taken to be one.

Then the phase representation of the pion scalar-isoscalar FF is

$$
\Gamma_{\pi}(t)=P_{n}(t) \exp \left[\frac{t}{\pi} \int_{4}^{\infty} \frac{\delta_{0}^{0}\left(t^{\prime}\right)}{t^{\prime}\left(t^{\prime}-t\right)} d t^{\prime}\right],
$$

where $\delta_{0}^{0}(t)$ is now the $S$-wave isoscalar $\pi \pi$ scattering phase shift to be exactly equal to the parametrization (7) with the parameter $A_{1}$ to be identified with the S-wave isoscalar $\pi \pi$ scattering length $a_{0}^{0}$. The number of nonzero parameters in (7) and their numerical values are found in a fitting procedure of the most precise data on $\delta_{0}^{0}(t)$ with theoretical errors in Fig. 1 to be generated by Garcia-Kamiński-Peláez-Yndurain (GKPY) equations [3] for the S-wave isoscalar $\pi \pi$ scattering amplitude.

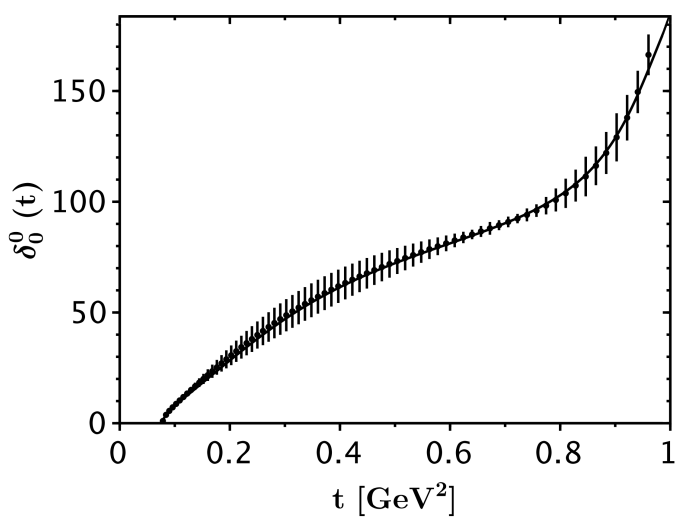

Figure 1. The data on $\delta_{0}^{0}(t)$ with theoretical errors to be generated by GKPY equations for the Swave isoscalar $\pi \pi$ scattering amplitude. Solid line represents our optimal fit of data with 5 nonzero coefficients $A_{i}$ in (7)

The data in Fig. 1 have been analyzed by the relation (7) up to the moment, when the minimum of $\chi^{2} /$ ndf was achieved. The latter has been found with the first 5 nonzero coefficients $A_{i}$ of the values

$$
\begin{array}{llr}
A_{1} & = & 0.2219 \pm 0.0029 \\
A_{2} & = & -0.0764 \pm 0.0423 \\
A_{3} & = & 0.1390 \pm 0.0251 \\
A_{4} & = & -0.0062 \pm 0.0053 \\
A_{5}= & -0.0135 \pm 0.0020
\end{array}
$$

and the final form of the $\mathrm{S}$-wave isoscalar $\pi \pi$ scattering phase shift $\delta_{0}^{0}(t)$ takes the form

$$
\delta_{0}^{0}(t)=\arctan \frac{A_{1} q+A_{3} q^{3}+A_{5} q^{5}}{1+A_{2} q^{2}+A_{4} q^{4}} .
$$


Substitution of (11) into (10), however, leads to the expression which does not allow one to calculate the corresponding integral explicitly.

Therefore we have used the equivalent form

$$
\delta_{0}^{0}(t)=\frac{1}{2 i} \ln \left[\frac{\left(1+A_{2} q^{2}+A_{4} q^{4}\right)+i\left(A_{1} q+A_{3} q^{3}+A_{5} q^{5}\right)}{\left(1+A_{2} q^{2}+A_{4} q^{4}\right)-i\left(A_{1} q+A_{3} q^{3}+A_{5} q^{5}\right)}\right]
$$

to (11) to be valid in the theory of functions of complex variable.

The latter leads to

$$
\Gamma_{\pi}(t)=P_{n}(t) \exp \left[\frac{\left(q^{2}+1\right)}{2 \pi i} \int_{-\infty}^{\infty} \frac{q^{\prime} \ln \frac{\left(1+A_{2} q^{\prime 2}+A_{4} q^{\prime 4}\right)+i\left(A_{1} q^{\prime}+A_{3} q^{\prime 3}+A_{5} q^{\prime 5}\right)}{\left(1+A_{2} q^{\prime 2}+A_{4} q^{4}\right)-i\left(A_{1} q^{\prime}+A_{3} q^{\prime 3}+A_{5} q^{\prime 5}\right)}}{\left(q^{\prime 2}+1\right)\left(q^{\prime 2}-q^{2}\right)} d q^{\prime}\right]
$$

and the integral in (13)

$$
I=\int_{-\infty}^{\infty} \frac{q^{\prime} \ln \frac{\left(q^{\prime}-q_{1}\right)\left(q^{\prime}-q_{2}\right)\left(q^{\prime}-q_{3}\right)\left(q^{\prime}-q_{4}\right)\left(q^{\prime}-q_{5}\right)}{\left(q^{\prime}-q_{1}^{*}\right)\left(q^{\prime}-q_{2}^{*}\right)\left(q^{\prime}-q_{3}^{*}\right)\left(q^{\prime}-q_{4}^{*}\right)\left(q^{\prime}-q_{5}^{*}\right)}}{\left(q^{\prime}+i\right)\left(q^{\prime}-i\right)\left(q^{\prime}+i b\right)\left(q^{\prime}-i b\right)} d q^{\prime}
$$

with

$$
q^{2}<0 \text { i.e. } q=i \sqrt{\frac{4-t}{4}} \equiv i b
$$

and

$$
\begin{array}{lllll}
q_{1}= & 0.00 & -i 2.0430 \pm 0.2029, & q_{1}^{*}=-q_{1}, \\
q_{2}= & 1.41470 \pm 0.0579 & +i 1.0749 \pm 0.0162, & q_{2}^{*}=-q_{3}, \\
q_{3}= & -1.4147 \pm 0.0579 & +i 1.0749 \pm 0.0162, & q_{3}^{*}=-q_{2}, \\
q_{4}= & 3.3827 \pm 0.0115 & +i 0.1744 \pm 0.0340, & q_{4}^{*}=-q_{5}, \\
q_{5}= & -3.3827 \pm 0.0115 & +i 0.1744 \pm 0.0340, & & q_{5}^{*}=-q_{4}
\end{array}
$$

now can be calculated in the framework of the theory of residue explicitly.

In order to carry out it practically, it is convenient to decompose the integral (14) into a sum of two integrals

$$
\begin{aligned}
I=I_{1}+I_{2}= & \int_{-\infty}^{+\infty} \frac{q^{\prime} \ln \frac{\left(q^{\prime}-q_{2}\right)\left(q^{\prime}-q_{3}\right)\left(q^{\prime}-q_{4}\right)\left(q^{\prime}-q_{5}\right)}{\left(q^{\prime}-q_{1}^{*}\right)}}{\left(q^{\prime}+i\right)\left(q^{\prime}-i\right)\left(q^{\prime}+i b\right)\left(q^{\prime}-i b\right)} d q^{\prime}+ \\
& \left(-\int_{+\infty}^{-\infty} \frac{q^{\prime} \ln \frac{\left(q^{\prime}-q_{1}\right)}{\left(q^{\prime}-q_{2}^{*}\right)\left(q^{\prime}-q_{3}^{*}\right)\left(q^{\prime}-q_{4}^{*}\right)\left(q^{\prime}-q_{5}^{*}\right)}}{\left(q^{\prime}+i\right)\left(q^{\prime}-i\right)\left(q^{\prime}+i b\right)\left(q^{\prime}-i b\right)} d q^{\prime}\right)
\end{aligned}
$$

according to singularities to be placed in the upper or lower half $q$-plane, as it is sketched in Fig. 2.

Then the explicit form of

$$
I=\frac{2 \pi i}{\left(q^{2}+1\right)} \ln \left(\frac{\left(q-q_{1}^{*}\right)}{\left(q-q_{2}^{*}\right)\left(q-q_{3}^{*}\right)\left(q-q_{4}^{*}\right)\left(q-q_{5}^{*}\right)} \frac{\left(i-q_{2}^{*}\right)\left(i-q_{3}^{*}\right)\left(i-q_{4}^{*}\right)\left(i-q_{5}^{*}\right)}{\left(i-q_{1}^{*}\right)}\right)
$$

is obtained in the straightforward way, if in the case of the first integral

$$
\oint \phi_{1}\left(q^{\prime}\right) d q^{\prime}=2 \pi i \sum_{n=1}^{2} \operatorname{Res}_{n}
$$




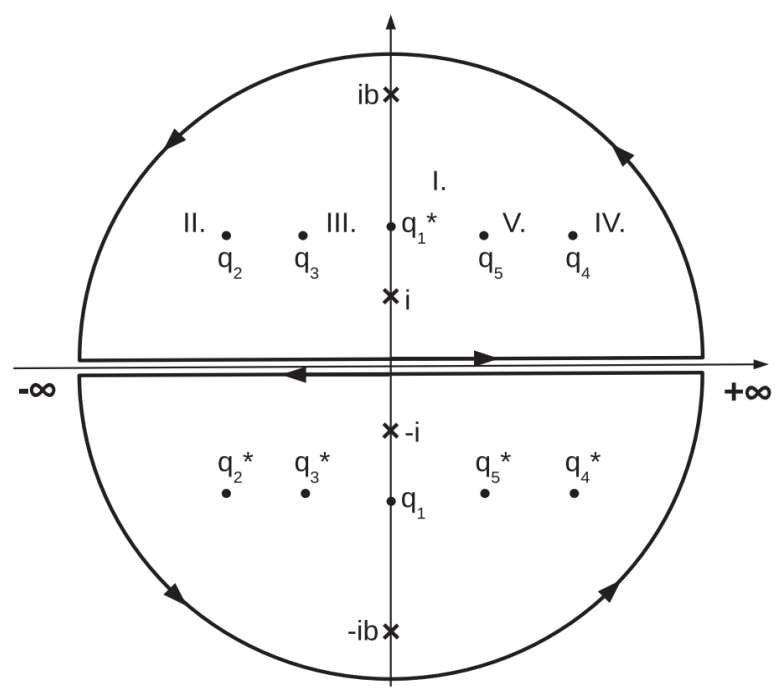

Figure 2. Poles $(\times)$ and branch points $(\bullet)$ of the integrands $\phi_{1}\left(q^{\prime}\right)$ and $\phi_{2}\left(q^{\prime}\right)$ with contours of integrations in the upper and the lower half $q$-planes, respectively

the contour of integration is closed in the lower half $q$-plane and in the second integral

$$
\oint \phi_{2}\left(q^{\prime}\right) d q^{\prime}=2 \pi i \sum_{n=1}^{2} \operatorname{Res}_{n}
$$

the contour of integration is closed in the upper half $q$-plane (see Fig. 2).

In a such way one avoids complicated calculations of the cut-contributions to be automatically generated by branch points under logarithms, if the contours are drawn as it is demonstrated in Fig. 2.

The substitution of (17) into (13) leads to the explicit form of the pion scalar-isoscalar FF

$$
\Gamma_{\pi}(t)=P_{n}(t) \frac{\left(q-q_{1}^{*}\right)}{\left(q-q_{2}^{*}\right)\left(q-q_{3}^{*}\right)\left(q-q_{4}^{*}\right)\left(q-q_{5}^{*}\right)} \frac{\left(i-q_{2}^{*}\right)\left(i-q_{3}^{*}\right)\left(i-q_{4}^{*}\right)\left(i-q_{5}^{*}\right)}{\left(i-q_{1}^{*}\right)},
$$

where $P_{n}(t)$ is any polynomial normalized at $t=0$ to one, however, it has not violate the asymptotic behavior of the pion scalar-isoscalar FF.

The pole $q=q_{3}^{*}$ on the second Riemann sheet in $t$-variable corresponds to the $f_{0}(500)$ meson resonance, now with the mass and the width, $m_{\sigma}=(459 \pm 29) \mathrm{MeV}$ and $\Gamma_{\sigma}=(517 \pm$ 77) $\mathrm{MeV}$ [7], respectively, which are compatible with the parameters obtained in [4, 5].

The Rev. Part. Physics (2016) [6] gives parameters of $f_{0}(500)$ to be $m_{\sigma}=(400-$ 550) $\mathrm{MeV}$ and $\Gamma_{\sigma}=(400-700) \mathrm{MeV}$.

A behavior of the $\Gamma_{\pi}(t)(20)$ at $-3 \mathrm{GeV}^{2}<t<3 \mathrm{GeV}^{2}$ is presented in Fig. 3 .

\section{Vector pion electromagnetic form factor and $\rho^{0}(770)$ meson parameters}

The vector isovector charged pion electromagnetic (EM) form factor $F_{\pi}^{E M, I=1}(t)$ is defined by the matrix element of the pion EM current $J_{\mu}^{E M}$ as follows

$$
\left\langle p_{2}\left|J_{\mu}^{E M}(0)\right| p_{1}\right\rangle=e F_{\pi}^{E M, I=1}(t)\left(p_{1}+p_{2}\right)_{\mu},
$$




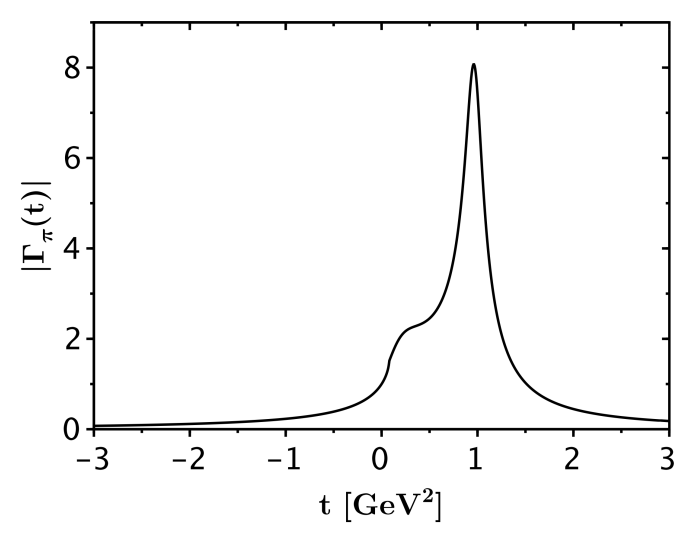

Figure 3. Behavior of the pion scalar form factor (20) with one zero and four poles at $-3 \mathrm{GeV}^{2}<t<$ $3 \mathrm{GeV}^{2}$. Results correspond to a fit to the output phase shifts from [3] by 5 free parameters. Physically may be interpreted region only below $990 \mathrm{MeV}\left(t \approx 0.98 \mathrm{GeV}^{2}\right)$

with $e$ to be the electric charge and $t=\left(p_{2}-p_{1}\right)^{2}$ the momentum transfer squared. The $F_{\pi}^{E M, I=1}(t)$ also fulfils all properties of the function $F(t)$ defined in Sect. 1, including the normalization $F_{\pi}^{E M, I=1}(0)=1$, if the electric charge is put to be one. Then $F_{\pi}^{E M, I=1}(t)$ can be represented by the phase representation

$$
F_{\pi}^{E M, I=1}(t)=P_{n}(t) \exp \left[\frac{t}{\pi} \int_{4}^{\infty} \frac{\delta_{1}^{1}\left(t^{\prime}\right)}{t^{\prime}\left(t^{\prime}-t\right)} d t^{\prime}\right],
$$

where $\delta_{1}^{1}(t)$ is now the $\mathrm{P}$-wave isovector $\pi \pi$ scattering phase shift and $P_{n}(t)$ is polynomial normalized at $t=0$ to one, however, it has not violate the asymptotic behavior of the charged pion EM FF.

In this case the model independent parametrization (7), taking into account a threshold behavior of the $\delta_{1}^{1}(t)_{|q| \rightarrow 0} \sim a_{1}^{1} q^{3}$, has to be adapted to the form

$$
\delta_{1}^{1}(t)=\arctan \frac{A_{3} q^{3}+A_{5} q^{5}+A_{7} q^{7}+\ldots}{1+A_{2} q^{2}+A_{4} q^{4}+A_{6} q^{6}+\ldots},
$$

where the parameter $A_{3}$ is identified with the P-wave isovector $\pi \pi$ scattering length $a_{1}^{1}$.

The number of nonzero parameters in (23) and their numerical values are again found in a fitting procedure of the most precise data on $\delta_{1}^{1}(t)$ with theoretical errors in Fig. 4 to be generated by Garcia-Kamiński-Peláez-Yndurain equations [3] for the P-wave isovector $\pi \pi$ scattering amplitude.

The minimum of $\chi^{2} /$ ndf was achieved by the first 4 nonzero values of coefficients in (23)

$$
\begin{aligned}
A_{2} & =0.1070 \pm 0.0329 \\
A_{3} \equiv a_{1}^{1} & =0.0321 \pm 0.0008 \\
A_{4} & =-0.03825 \pm 0.0030 \\
A_{5} & =0.0003 \pm 0.0002
\end{aligned}
$$




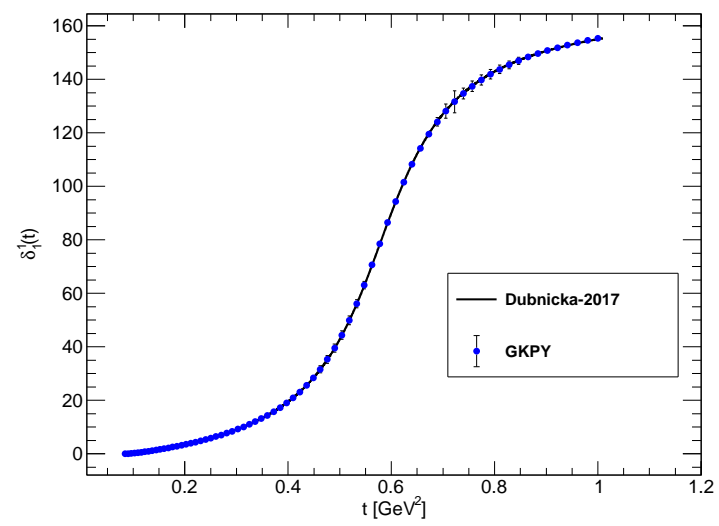

Figure 4. Optimal description of the GKPY $\delta_{1}^{1}(t)$ data. Solid line represents our optimal fit of data with 4 nonzero coefficients $A_{i}$ in (23)

and the roots of the corresponding polynomials in the numerator and denominator of the equivalent to (23) logarithmic relation

$$
\delta_{1}^{1}(t)=\frac{1}{2 i} \ln \left[\frac{\left(1+A_{2} q^{2}+A_{4} q^{4}\right)+i\left(A_{3} q^{3}+A_{5} q^{5}\right)}{\left(1+A_{2} q^{2}+A_{4} q^{4}\right)-i\left(A_{3} q^{3}+A_{5} q^{5}\right)}\right]
$$

are

$$
\begin{array}{lcccl}
q_{1}= & -2.5480 \pm 0.0020 & +i 0.2752 \pm 0.0016, & & q_{1}^{*}=-q_{2}, \\
q_{2}= & 2.5480 \pm 0.0020 & +i 0.2752 \pm 0.0016, & q_{2}^{*}=-q_{1} \\
q_{3}= & 0.0 & -i 1.8432 \pm 0.0658, & q_{3}^{*}=-q_{3} \\
q_{4}= & 0.0 & +i 2.146 \pm 0.1054, & q_{4}^{*}=-q_{4} \\
q_{5}= & 0.0 & -i 139.793 \pm 0.152542, & q_{5}^{*}=-q_{5} .
\end{array}
$$

A substitution of (25) with (26) into (22) leads to

$$
F_{\pi}^{E M, I=1}(t)=P_{n}(t) \exp \left[\frac{\left(q^{2}+1\right)}{2 \pi i} \int_{-\infty}^{\infty} \frac{\left(q^{\prime}-q_{1}\right)\left(q^{\prime}-q_{2}\right)\left(q^{\prime}-q_{3}\right)\left(q^{\prime}-q_{4}\right)\left(q^{\prime}-q_{5}\right)}{\left(q^{\prime}-q_{1}^{*}\right)\left(q^{\prime}-q_{2}^{*}\right)\left(q^{\prime}-q_{3}^{*}\right)\left(q^{\prime}-q_{4}^{*}\right)\left(q^{\prime}-q_{5}^{*}\right)} d q^{\prime}\right]
$$

and the integral in (27) is calculated in the same way as it was done in the case of the scalarisoscalar pion $\mathrm{FF}$.

The pole $q=q_{1}^{*}$ on the second Riemann sheet in $t$-variable corresponds to the $\rho^{0}(770)$ meson resonance, with the mass and the width, $m_{\rho}=(763.56 \pm 0.51) \mathrm{MeV}$ and $\Gamma_{\rho}=(143.09 \pm$ 0.82) $\mathrm{MeV}$ [7], respectively.

The Rev. Part. Physics (2016) [6] gives parameters of $\rho^{0}(770)$ to be $m_{\rho}=(775.26 \pm$ $0.25) \mathrm{MeV}$ and $\Gamma_{\rho}=(149.1 \pm 0.8) \mathrm{MeV}$.

\section{Conclusions}

Taking into account phase representations with one subtraction of the pion scalar-isoscalar form factor and vector-isovector charged pion electromagnetic form factor and exploiting the 
most accurate up to now information on the S-wave isoscalar and the P-wave isovector $\pi \pi$ scattering phase shifts, respectively, to be obtained from the existing inaccurate experimental data by means of the Garcia-Kamiński-Peláez-Yndurain Roy-like equations with an imposed crossing symmetry condition, in the framework of the so-called "fully solvable mathematical scheme" the most reliable values of the $f_{0}(500)$ and $\rho^{0}(770)$ meson mass and width are determined.

The work was supported by VEGA grant No.2/0153/17.

\section{References}

[1] S. Dubnicka, A.Z. Dubnickova and A. Liptaj, Phys. Rev. D 90, 114003 (2014)

[2] J. Gasser and U-G. Meissner, Nucl. Phys. B 357, 90 (1991)

[3] R. Garcia-Martin, R. Kamiński, J.R. Pela'ez, J. Ruiz de Elvira and F.J. Yndurain, Phys. Rev. D 83, 074004 (2011)

[4] I. Caprini, G. Colangelo and H. Leutwyler, Phys. Rev. Lett. 96, 132001 (2006)

[5] R. Garcia-Martin, R. Kamiński, J. R.Pela'ez and J. Ruiz de Elvira, Phys. Rev. Lett. 107, 072001 (2011)

[6] C. Patrignani et al. [Particle Data Group], Review of Particle Physics, Chin. Phys. C 40, 100001 (2016)

[7] S. Dubnicka, A.Z. Dubnickova, R. Kamiński and A. Liptaj, Phys. Rev. D 94, 054036 (2016) 